\title{
Line Ratio Imaging of a Gas Discharge
}

\author{
D. Samsonov and J. Goree, Member, IEEE
}

\begin{abstract}
The intensity ratio of two atomic or ionic spectral lines in a plasma is a function of electron temperature. By imaging two spectral lines, and computing the line ratio, pixel by pixel, an image of the electron temperature is produced. This is demonstrated using a dusty plasma consisting of submicron particles suspended in an argon RF glow discharge.
\end{abstract}

Index Terms-Discharge, dusty plasma, electron temperature, imaging, line ratio, particles, particulates, spectroscopy.

$\mathbf{T}$ HE intensity ratio of two spectral lines is a common way of making nonperturbing measurements of the electron temperature $T_{e}$ [1], [2]. Generally this method has been used to make single point measurements. Recently, however, Meo et al. [3] imaged D and He spectral lines to produce line ratio images in a tokamak plasma.

Here we demonstrate line ratio imaging in a lowtemperature argon discharge. We chose a dusty plasma, i.e., a plasma containing small particles, because it often has a so-called "void" region [4] with a strongly enhanced glow [5]. We used this imaging method to determine whether the enhanced glow is due to a higher $T_{e}$ or a higher electron density $n_{e}$.

In this paper, the key assumption is a monotonic dependence of $T_{e}$ with the line ratio. This allows us to identify qualitatively where in the discharge the electrons are hottest. We do not adopt a specific atomic physics model in order to quantify $T_{e}$, although one could do this. The corona model, for example, predicts a monotonic variation of the line ratio with $T_{e}$ [6], [7]. This model assumes an optically-thin plasma, a low population of excited states, excitation by electron impact, and de-excitation by radiative decay. It also assumes a Maxwellian electron distribution function. When the distribution is not Maxwellian (likely the case in our experiment) the method yields an effective $T_{e}$ for the portion of the electron distribution in the energy range of the two excited states that are probed. The corona model neglects stepwise excitation via metastable states (which can be important for high-density discharges), population by cascade from upper levels, and quenching by collisions with neutral atoms. When these conditions are satisfied, it is possible to measure $T_{e}$ quantitatively, provided that certain atomic parameters can be computed. Otherwise one can use the line ratio as a qualitative indicator of $T_{e}$, as we do here. An alternative is to use a more general model

Manuscript received July 15, 1998; revised September 11, 1998. This work was supported by the National Science Foundation and NASA.

D. Samsonov is with the Max Planck Institut für extraterrestrishe Physik, 85740 Garching, Germany.

J. Goree is with the Department of Physics and Astronomy, University of Iowa, Iowa City, Iowa 52242 USA (e-mail:

Publisher Item Identifier S 0093-3813(99)02570-9.

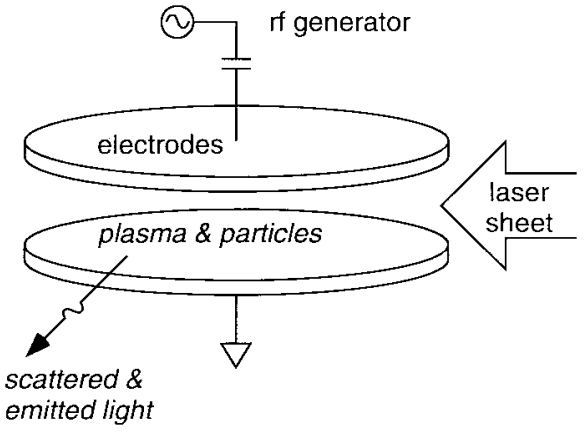

(a)

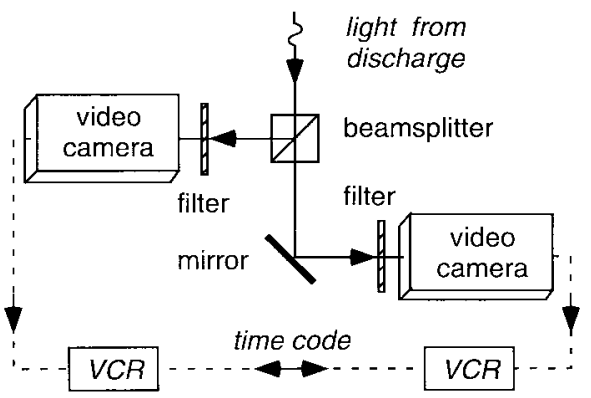

(b)

Fig. 1. (a) Discharge schematic. Particles grown in the capacitively-coupled RF discharge are illuminated by a vertical sheet of laser light. (b) Optical configuration. Two video cameras view the same area through a beam splitter. Optical bandpass filters select the spectral lines.

[8], which includes two-step excitation and is free from the restrictive assumptions of the corona model.

A parallel-plate RF discharge, Fig. 1, was formed by applying $100 \mathrm{~W}$ of $13.56 \mathrm{MHz}$ power to the upper electrode through a matching network and a coupling capacitor. The lower electrode was grounded. The electrodes $(8 \mathrm{~cm}$ diameter and $2 \mathrm{~cm}$ separation) were covered with graphite sputtering targets, which yielded a carbon vapor that condensed in the gas phase to produce particles up to $350 \mathrm{~nm}$ diameter. The Ar gas flow was $60 \mathrm{sccm}$, at 400 mtorr pressure.

The discharge area was imaged by two video cameras viewing the same area through a beam splitter and 10-nm bandpass interference filters. Video was recorded synchronously on two VCR's and then digitized by an 8-bit frame grabber. The cameras were calibrated relative to one another by taking into account the optical throughput of the filters, beam splitter, and the charge-coupled device sensitivity, all at the measured wavelength. We also calibrated the output signal of the cameras versus optical intensity, yielding sensitivity curves that inherently take into account the so-called $\gamma$ correction (the nonlinear response of a video camera to the optical intensity). The line ratio was calculated, pixel-by-pixel, to synthesize false-color images. 

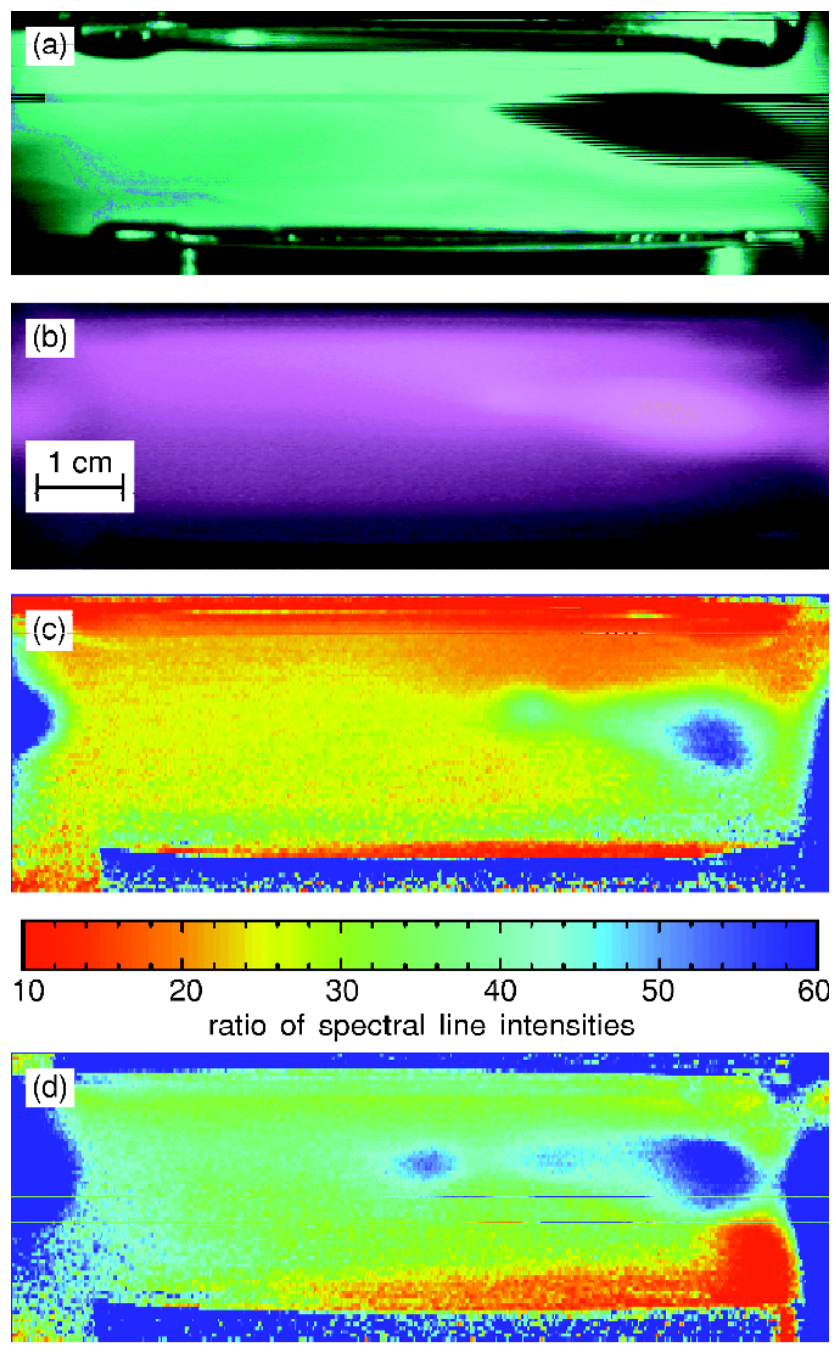

Fig. 2. Images of the interelectrode region. Between the two electrodes, seen at the top and bottom, is the dusty Ar plasma. (a) Laser light scattering reveals a particle cloud that has a void on the right side. A lighter color corresponds to a higher particle number density. (b) Discharge glow from ArI at 763.5 $\mathrm{nm}$. The glow is enhanced in the void. (c) Line ratio image for ArI/ArI (763.5 $\mathrm{nm} / 549.5 \mathrm{~nm}$ ). (d) Line ratio image for ArI/ArII $(763.5 \mathrm{~nm} / 488 \mathrm{~nm})$. Both (c) and (d) have the same color scaler here where a smaller line ratio (red) corresponds to higher $T_{e}$. The void has a lower $T_{e}$ than in the surrounding plasma.

The particle cloud, Fig. 2(a), was imaged using a vertical sheet of argon laser light and a $488 \mathrm{~nm}$ filter, and the discharge glow, Fig. 2(b), was imaged using the $763.511 \mathrm{~nm}$ ArI line. Both of these images show a distinct feature on the right side. This is a void in the particle cloud, which is accompanied by an intense glow [5].

We used line ratio imaging to determine whether the intense glow was due to an enhanced $T_{e}$ or a higher $n_{e}$. Fig. 2(c) is a line ratio image for the $763.511 \mathrm{~nm}$ ArI and $549.587 \mathrm{~nm}$ ArI lines, corresponding to $4 p[1 / 2]-4 s[3 / 2]^{\circ}$ and $6 d[7 / 2]-$ $4 p[5 / 2]$ transitions, with upper levels at 13.17 and $15.33 \mathrm{eV}$, respectively. Interpreting this image, we see that compared to the surrounding dusty plasma, the void has a smaller $T_{e}$ (or more precisely, since the electrons are probably not Maxwellian, a smaller effective $T_{e}$ in the energy range $13-15$ $\mathrm{eV})$.

As a test, we also prepared another line ratio image for $763.511 \mathrm{~nm}$ ArI and $487.986 \mathrm{~nm}$ ArII lines, where the ArII line corresponds to the $4 p^{2} D^{\mathrm{o}}-4 s^{2} P$ transition. This yielded Fig. 2(d), which appears similar to the ArI/ArI image in Fig. 2(c). Unlike ArI/ArI, the ArI/ArII ratio also depends on $n_{e}$. The ArI/ArI ratio is a function of only $T_{e}$ because ArI line intensities depend on $n_{e}$ in the same way and this cancels out. On the contrary, the ArI/ArII ratio decreases with $n_{e}$ because the intensity of ArII line is proportional to the ion density $n_{i}$, which is produced by electron impact ionization. If both line ratios are measured simultaneously, which was not done for this paper, then it is possible to calculate $n_{i}$ and $n_{e}$, provided the ionization cross section is known.

In this experiment, our imaging method revealed that the enhanced glow in the void of the dust particle cloud is not due to enhanced electron temperature, so that it must be instead due to a higher electron density. This is consistent with the tendency of dust particles to deplete electrons and thus reduce electron density in the dust cloud. Further details of the dusty plasma experiment are presented elsewhere [4], [5]. Additional images and videos from the experiment can be found on our web server [9].

\section{ACKNOWLEDGMENT}

The authors would like to thank G. Morfill for research facilities in Garching.

\section{REFERENCES}

[1] H. R. Griem, Plasma Spectroscopy. New York: McGraw-Hill, 1964.

[2] R. W. P. McWhirter, "Spectral intensities," in Plasma Diagnostic Techniques, R. H. Huddlestone and S. L. Leonard, Eds. New York: Academic, 1965.

[3] F. Meo, B. L. Stansfield, M. Chartré, P. de Villers, R. Mar.and, and G. Ratel, "Spectroscopic imaging system for quantitative analysis of the divertor plasma of the tokamak de varennes," Rev. Sci. Instrum., vol. 68, no. 9, pp. 3426-3435, 1997.

[4] G. Praburam and J. Goree, "Experimental observation of very lowfrequency macroscopic modes in a dusty plasma," Phys. Plasmas, vol. 3, no. 4, pp. 1212-1219, 1996.

[5] D. Samsonov and J. Goree, "Instabilities in a dusty plasma with ion drag and ionization," Phys. Rev. E, vol. 59, no. 1, pp. 1047-1058, 1999.

[6] R. L. Rhoades and S. M. Gorbatkin, "Characterization of ar/cu electroncyclotron-resonance plasmas using optical emission spectroscopy," $J$. Appl. Phys., vol. 80, no. 1, pp. 2605-2613, 1994.

[7] T. M. Desai, S. V. Gogawale, A. B. Shukla, N. K. Joshi, U. S. Salgaonkar, and G. L. Bhale, "Electron temperature measurements in uhv systems by spectrocopic and Langmuir probe techniques," Vacuum, vol. 46, no. 3, pp. 223-226, 1995.

[8] M. V. Malyshev and V. M. Donnelly, "Determination of electron temperatures in plasmas by multiple rare gas optical emission, and implications for advanced actinometry," J. Vac. Sci. Technol. A, vol. 15, no. 3, pp. 550-558, 1997.

[9] D. Samsonov and J. Goree, "Video of an ionization instability in a dusty plasma," [Online]. Available www: http://dusty.physics.uiowa.edu/Movies/Movie_index.html, other images are at http://dusty.physics.uiowa.edu. 